\title{
Effects of luteolin on regulatory proteins and enzymes for myocyte calcium circulation in hypothermic preserved rat heart
}

\author{
QINGFENG YAN ${ }^{1}$, YUEPING LI ${ }^{1}$, JIA YAN ${ }^{2}$, YING ZHAO $^{3}$, YUNZHONG LIU $^{3}$ and $\mathrm{SU} \mathrm{LIU}^{3}$ \\ ${ }^{1}$ Department of Basic Medical and Life Science, Hainan Medical College, Haikou, Hainan 571101; \\ ${ }^{2}$ Department of Life Science and Ecology, Hainan Tropical Ocean College, Sanya, Hainan 572022; \\ ${ }^{3}$ Department of Cardiac Surgery, The Affiliated Hospital of Hainan Medical College, Haikou, Hainan 571101, P.R. China
}

Received April 25, 2017; Accepted September 9, 2017

DOI: $10.3892 /$ etm.2017.5514

\begin{abstract}
Heart transplantation has been applied in the clinic as an optimal solution for patients with end stage cardiac failure for a number of years. However, hypothermic preservation of the heart remains limited to 4-6 $\mathrm{h}$ and calcium accumulation over time is an important factor resulting in cell death. To provide longer and safer storage for donor hearts, it was demonstrated in our previous study that luteolin, a traditional Chinese medicine used to treat cardiovascular diseases, inhibits cell death and L-type calcium currents during hypothermic preservation. In the current study, the protective role of luteolin in modulating cardiomyocyte calcium cycling was further investigated. Intracellular calcium overload has already been implicated in hypothermia-induced dysfunction of cardiomyocytes. University of Wisconsin (UW) solution supplemented with $7.5,15$ or $30 \mu \mathrm{mol} / 1$ luteolin was used to preserve fresh isolated cardiomyocytes at $4{ }^{\circ} \mathrm{C}$. The results demonstrated that all three doses of luteolin supplementation attenuated calcium overload over a $6 \mathrm{~h}$ preservation period. Luteolin also suppressed the accumulation of important regulatory proteins and enzymes for cardiomyocyte calcium circulation, mitochondria $\mathrm{Ca}^{2+}$ uniporter and calmodulin, which are normally induced by cold storage in UW solution. Protein Kinase A activity was also suppressed in cardiomyocytes preserved in luteolin supplemented UW solution, while $\mathrm{Ca}^{2+}-\mathrm{Mg}^{2+}$-ATPase activity was increased. The results demonstrated that luteolin confers a cardioprotective effect through inhibiting the changes of calcium regulators during cold storage and therefore ameliorates $\mathrm{Ca}^{2+}$ overload in rat cardiomyocytes.
\end{abstract}

Correspondence to: Professor Yueping Li, Department of Basic Medical and Life Science, Hainan Medical College, 3 Xueyuan Road, Haikou, Hainan 571101, P.R. China

E-mail: hkwz1001@gmail.com

Key words: cardiomyocyte, luteolin, calcium, hypothermic preservation

\section{Introduction}

Heart transplantation (HT) is the most effective treatment for patients with congenital heart disease without suitable alternative treatment options or end-stage heart failure (1,2). Although the demand for HT has increased by $130 \%$ over past decades, the number of transplants performed every year increased by $5.2 \%$ due to limited organ donation and short-time preservation of the heart (3). Cold ischemic storage of donor hearts is restricted to 4-6 h $(4,5)$, which limits transplantations depending on emergent circumstances and the distance of the donor heart from the potential recipient. Extension of heart preservation may therefore greatly benefit patients waiting for a heart transplantation.

Luteolin (3',4',5,7-tetrahydroxyflavone) is a flavone with various pharmacological activities, including scavenging for oxygen free radicals, inhibiting lipid peroxidation, antithrombotic activity and anti-inflammatory responses $(6,7)$. It also has a protective role in ischemia-reperfusion injury by suppressing apoptosis of cardiomyocytes $(8,9)$. As a good candidate for a heart preservation additive, our previous study demonstrated that luteolin enhanced cardiac contractile and diastolic function and coronary flow of rat hearts compared with the control group when used as an additive to University of Wisconsin (UW) solution (10). Luteolin also reduced the L-type calcium current of rat ventricular myocytes in a dose-dependent manner (9). Therefore, it was hypothesized that luteolin may modulate calcium ion homeostasis in order to prolong heart preservation.

$\mathrm{Ca}^{2+}$ homeostasis serves a critical role in maintaining cardiomyocyte function. Intracellular $\mathrm{Ca}^{2+}$ overload may lead to cell death and increased reactive oxygen species (ROS) (11). As a primary transporter for mitochondria $\mathrm{Ca}^{2+}$ influx, mitochondria $\mathrm{Ca}^{2+}$ uniporter $(\mathrm{MCU})$ resides in the inner membrane of mitochondria and allows the cytosolic $\mathrm{Ca}^{2+}$ to be transported into mitochondria (12). Inhibition of MCU has been demonstrated to prevent mitophagy in neurocytes through modulating $\mathrm{Ca}^{2+}$ influx into mitochondria, suggesting low MCU concentration helps maintain mitochondrial morphology and function (13). Protein kinase A (PKA) is a cyclic adenosine monophosphate (cAMP)-dependent protein kinase and regulates L-type voltage-operated calcium channels and intracellular calcium channels by activation of 
$\beta$-adrenergic receptor $(\beta$-AR) $(14,15)$. Calmodulin $(\mathrm{CaM})$ is an intracellular $\mathrm{Ca}^{2+}$ sensor, which also serves an indispensible role in intracellular $\mathrm{Ca}^{2+}$ cycling. It transduces intracellular $\mathrm{Ca}^{2+}$ signaling following the binding of $\mathrm{Ca}^{2+}$ and $\mathrm{CaM}$, the product of which is capable of regulating cardiomyocyte excitation-contraction coupling through its binding partner ryanodine receptors $(16,17) . \mathrm{Ca}^{2+}-\mathrm{Mg}^{2+}$-ATPase, as a calcium pump, regulates ATP-dependent calcium across the sarcoplasmic reticulum (SR) (18). Impairing $\mathrm{Ca}^{2+}-\mathrm{Mg}^{2+}-\mathrm{ATPase}$ activity may lead to increased SR calcium accumulation (19). Understanding the effect of luteolin on these intracellular $\mathrm{Ca}^{2+}$ regulator proteins may shed new light on the process of intracellular $\mathrm{Ca}^{2+}$ overload in cardiomyocytes during hypothermic preservation. In the current study, the effect of different doses of luteolin in UW solution on preserving rat cardiomyocytes was evaluated.

\section{Materials and methods}

Reagents. Luteolin (>99\% purity) was purchased from Hangzhou FST Pharmaceutical Co., Ltd. (Hangzhou, China). UW solution was purchased from Bristol-Myers Squibb (New York, NY, USA). Dulbecco's modified Eagle's medium (DMEM) solution, pentobarbital sodium and heparin were purchased from Sigma-Aldrich (Merck KGaA, Darmstadt, Germany). The calcium florescent detection kits for mitochondria (cat. no. HL10153.1) and sarcoplasmic reticulum (SR; cat. no. GMS10157.1), and intracellular calcium concentration fluorescent detection kits (cat. no. JM325) were purchased from Shanghai Haling Biological Technology Co., Ltd. (Shanghai, China). Fluo-3 acetoxymethyl (AM) was purchased from Beyotime Institute of Biotechnology (Haimen, China). Collagenase type I was purchased from Invitrogen; Thermo Fisher Scientific, Inc. (Waltham, MA, USA). Low $\mathrm{Ca}^{2+}$ enzymatic solution $(50 \mu \mathrm{mol} / \mathrm{l})$ was prepared by dissolving $20 \mathrm{mg}$ collagenase type 1, $2 \mathrm{mg}$ protease E (Sigma-Aldrich; Merck $\mathrm{KGaA}$ ), $10 \mathrm{mg}$ bovine serum albumin (MP Biomedicals, LLC, Santa Ana, CA, USA) and $72 \mathrm{mmol} / 1$ calcium chloride into $30 \mathrm{ml}$ calcium free Tyrode's solution, which was composed of $140 \mathrm{mM}$ sodium chloride, $5 \mathrm{mM}$ potassium chloride, $10 \mathrm{mM}$ HEPES, $2 \mathrm{mM}$ BAPTA, $10 \mathrm{mM}$ glucose, and $10 \mathrm{mM}$ Na pyruvate ( $\mathrm{pH} 7.4 \pm 0.2)$.

Animals. A total of 40 6-8 week old healthy male SD rats were purchased from Xi'an Jiaotong University Animal Center (Xi'an, China), weighing 250-300 g. Rats were housed in a pathogen-free environment (temperature, $20-30^{\circ} \mathrm{C}$; relative humidity, 45-60\%; lighting cycle, $12 \mathrm{~h} /$ day) and had ad lbitum access to food and drinking water. All animal experiments were performed according to the Guide of Care and Use of Laboratory Animals (20) and approved by the Animal Care and Use Committee of the Hainan Medical College (Haikou, China)

Heart perfusion and cardiomyocyte isolation. Following sacrifice, rat cardiomyocytes were isolated following a previously described protocol (21). In brief, $30 \mathrm{mg} / \mathrm{kg}$ pentobarbital sodium was intraperitoneally injected into the anesthetized rats, followed by $3 \mathrm{mg} / \mathrm{kg}$ heparin to prevent blood clot. Heart tissues were quickly isolated following a previously published protocol (10) and were then placed in calcium free Tyrode's solution at $4^{\circ} \mathrm{C}$. Isolated rat hearts were first perfused with oxygenated calcium free Tyrode's solution at $37^{\circ} \mathrm{C}$ until cardiac arrest using the Langendorff technique (22). A low $\mathrm{Ca}^{2+}$ enzymatic solution $(50 \mu \mathrm{mol} / \mathrm{l})$ was then used to further perfuse the rat hearts for 20-40 mins. When the hearts were softened, they were then again perfused with 180-200 $\mu \mathrm{mol} / \mathrm{l}$ low $\mathrm{Ca}^{2+}$ enzymatic solution for $5 \mathrm{~min}$. Following the $20 \mathrm{~min}$ perfusion process, ventricles were chopped and filtered with Krebs-Bicarbonate (KB) solution composed of $50 \mathrm{mM}$ L-glutamine, $20 \mathrm{mM}$ potassium chloride, $1 \mathrm{mM}$ HEPES, $10 \mathrm{mM}$ glucose, $80 \mathrm{mM}$ potassium hydroxide, $30 \mathrm{mM}$ dipotassium phosphate, $20 \mathrm{mM}$ taurine, $3 \mathrm{mM}$ magnesium sulfate and 0.5 mM EDTA (all Sigma-Aldrich; Merck KGaA) and incubated for $2 \mathrm{~h}$ at $37^{\circ} \mathrm{C}$. A total of $5 \times 10^{6}$ harvested cardiomyocytes were then washed with $\mathrm{KB}$ solution and preserved in $5 \mathrm{ml} \mathrm{UW}$ solution or DMEM. Luteolin was used to supplement UW solution at a low dose of $7.5 \mu \mathrm{mol} / 1$, middle dose of $15 \mu \mathrm{mol} / 1$ and a high dose of $30 \mu \mathrm{mol} / \mathrm{l}$. The groups used were as follows: i) A UW group, consisting of cardiomyocytes preserved in UW solution only; ii) a DMEM group, consisting of cardiomyocytes preserved in DMEM only; iii) a $7.5 \mu \mathrm{mol} / 1$ luteolin group, consisting of cardiomyocytes preserved in UW solution supplemented with $7.5 \mu \mathrm{mol} / 1$ luteolin; iv) a $15 \mu \mathrm{mol} / 1$ luteolin group, consisting of cardiomyocytes preserved in UW solution supplemented with $15 \mu \mathrm{mol} / 1$ luteolin; and v) a $30 \mu \mathrm{mol} / 1$ luteolin group, consisting of cardiomyocytes preserved in UW solution supplemented with $30 \mu \mathrm{mol} / 1$ luteolin.

Calcium florescence detection. To detect calcium florescence, $2 \mu \mathrm{M}$ Fluro-3 AM was incubated with cardiomyocytes for $1 \mathrm{~h}$ at $37^{\circ} \mathrm{C}$. Samples were washed twice with PBS and observed under a Multiphoton microscope (710 NLO; Carl Zeiss AG, Oberkochen, Germany) with an excitation wavelength of $490 \mathrm{~nm}$ and an emission wavelength of $525 \mathrm{~nm}$ to detect the level of $\mathrm{Ca}^{2+}$ in the cytosol. DAPI purchased from Wuhan Boster Biological Technology, Ltd. (Wuhan, China) was used for nuclear staining at room temperature for $5 \mathrm{mins}$. The same wavelengths were applied for the mitochondria and SR calcium detection kits, which were used according to the manufacturer's protocols. Calcium florescence intensity was analyzed semi-quantitatively by a trained examiner in a blinded manner, with four sections per animal.

ELISA. The levels of CaM and MCU in the cardiomyocytes were examined using a Rat CaM ELISA kit (cat. no. E-EL-M0611) from Elabscience Biotechnology Co., Ltd. (Houston, TX, USA) and Rat MCU ELISA kit (cat. no. ABIN1744117) from Blue Gene Biotech Co., Ltd. (Shanghai, China), respectively. PKA and $\mathrm{Ca}^{2+}-\mathrm{Mg}^{2+}$-ATPase activity were quantified by Rat protein kinase A (cat. no. ADI-EKS-390A) and $\mathrm{Ca}^{2+}-\mathrm{Mg}^{2+}$-ATPase ELISA kits (cat. no. A070-3) purchased from Enzo Life Sciences, Inc. (Farmingdale, NY, USA) and Nanjing Jiancheng Bioengineering Institute (Nanjing, China), respectively. Kits were used according to the manufacturers' protocols.

Statistical analysis. All data are presented as the mean \pm standard error of the mean. Two-way analysis of variance followed by Bonferroni's correction post hoc test was used for different preservation solutions and times to determine 


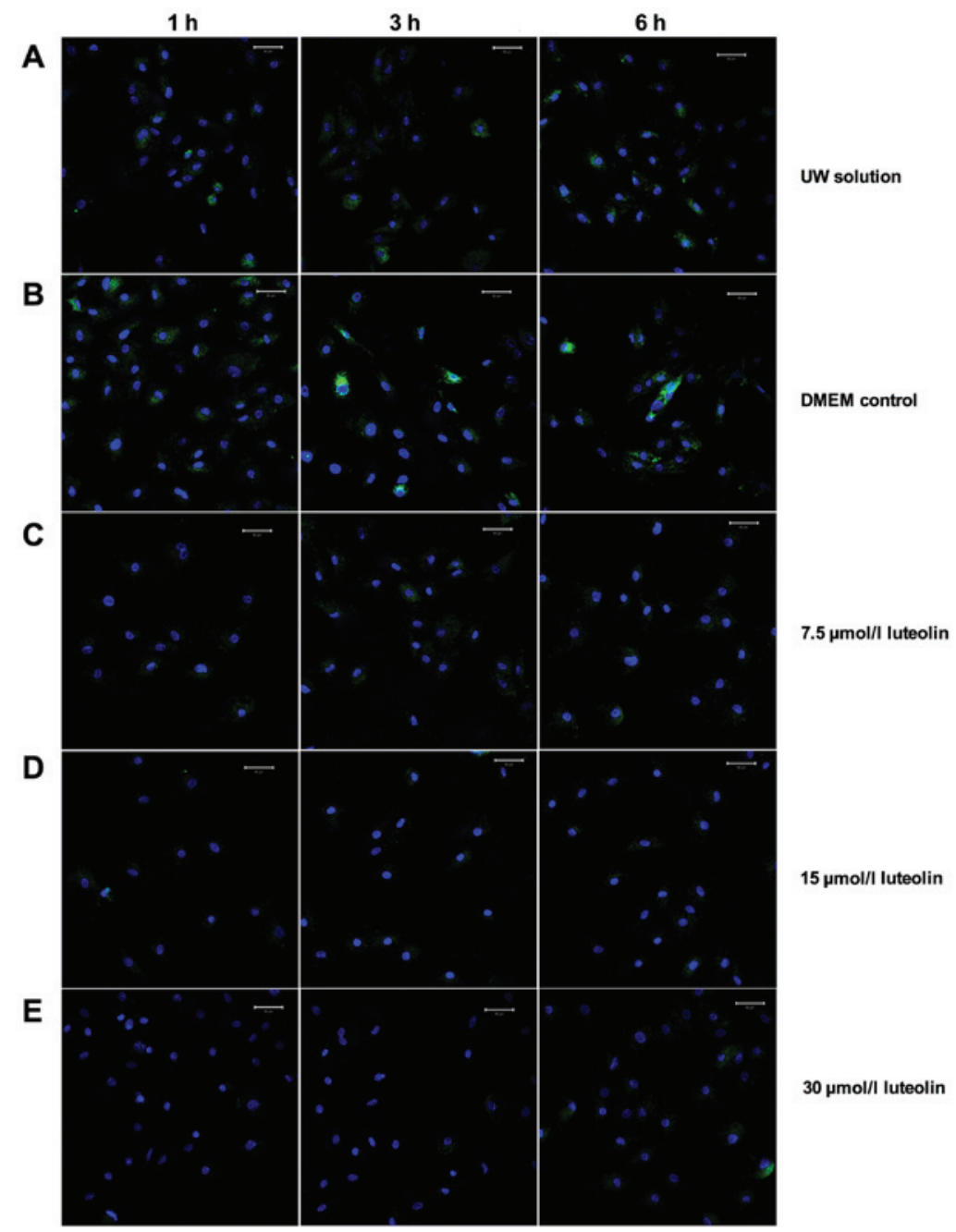

Figure 1. Luteolin attenuates cytosolic calcium accumulation. Multiphoton microscopy was applied to detect cytosolic $\mathrm{Ca}^{2+}$ in cardiomyocytes preserved in the following solutions: (A) UW solution; (B) DMEM; (C) $7.5 \mu \mathrm{mol} / 1$ luteolin supplemented UW solution; (D) $15 \mu$ mol/1 luteolin supplemented UW solution and (E) $30 \mu \mathrm{mol} / 1$ luteolin supplemented UW solution. Scale bar, $50 \mu \mathrm{m}$. Green fluorescence indicates Fluro-3 labeled $\mathrm{Ca}^{2+}$, while blue fluorescence indicates stained nuclei. UW solution, University of Wisconsin solution; DMEM, Dulbecco's modified Eagle's medium.

statistical differences between groups. All statistical analyses were performed using GraphPad Prism 6.0 for Macintosh (GraphPad Software, Inc., La Jolla, CA, USA). P $<0.05$ was considered to indicate a statistically significant difference.

\section{Results}

Luteolin attenuates intracellular calcium overload in rat cardiomyocytes during hypothermic preservation. To determine the effect of luteolin on modulating calcium ion cycling, 7.5, 15 and $30 \mu \mathrm{mol} / 1$ luteolin-supplemented UW solution was used to culture cardiomyocytes for $6 \mathrm{~h}$ and two control groups consisted of cardiomyocytes preserved in UW solution only or DMEM only. Following the labeling of cytosol free $\mathrm{Ca}^{2+}$ with a Fluro-3 AM fluorescence probe, the confocal microscopy images were captured following 1,3 and $6 \mathrm{~h}$ preservation (Fig. 1). The results demonstrated that universally accepted heart preservation solution, UW solution, had a similar capacity to restrain cytosol free calcium ion load compared with DMEM media (Figs. 1A and B, and 2). There was a significant reduction in cytosol $\mathrm{Ca}^{2+}$ in the three groups using different doses of luteolin to supplement UW solution throughout the $6 \mathrm{~h}$ preservation period compared with the UW solution control group (all $\mathrm{P}<0.01$; Figs. $1 \mathrm{C}-\mathrm{E}$ and 2). The $\mathrm{Ca}^{2+}$ concentration in the cytosol of cardiomyocytes following $1 \mathrm{~h}$ UW solution preservation was increased by $31 \%$ compared with those preserved with $7.5 \mu \mathrm{mol} / 1$ luteolin for $6 \mathrm{~h}$ (Fig. 2). The results therefore demonstrated that luteolin significantly decreases the cytosol $\mathrm{Ca}^{2+}$ concentration in cardiomyocytes during hypothermic preservation (all $\mathrm{P}<0.01$, Fig. 2).

Luteolin inhibits mitochondria calcium overload in rat cardiomyocytes during hypothermic preservation. It is known that calcium cycling in cardiomyocytes primarily occurs through calcium channels on the sarcolemma, which allows $\mathrm{Ca}^{2+}$ influx, SR involved intracellular calcium regulation and the transportation pathway of $\mathrm{Ca}^{2+}$ in mitochondria (18). A reduced $\mathrm{Ca}^{2+}$ presence was observed in the cytosol in the treatment groups, therefore, the influence of UW solution supplemented with luteolin on mitochondrial $\mathrm{Ca}^{2+}$ concentration and $\mathrm{SR} \mathrm{Ca}^{2+}$ concentration was investigated (Fig. 3). In the DMEM and UW solution control groups, calcium ions accumulated inside the mitochondria during the $6 \mathrm{~h}$ cold preservation period (Figs. 3A and B, and 4). The concentration of mitochondrial 


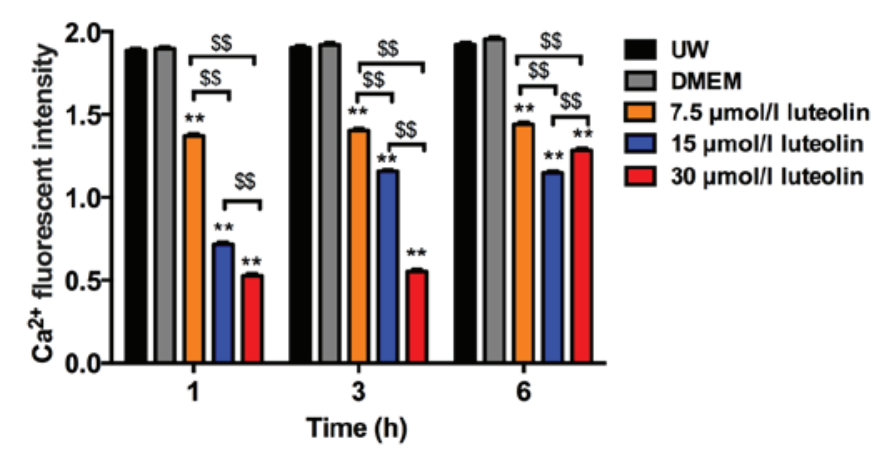

Figure 2. Fluorescence intensity of cytosolic $\mathrm{Ca}^{2+}$ in the different groups. Data are presented as the mean \pm standard error of the mean. Experiments were performed in triplicate. ${ }^{* *} \mathrm{P}<0.01$ vs. UW solution group; ${ }^{\$ \$} \mathrm{P}<0.01$. UW solution, University of Wisconsin solution; DMEM, Dulbecco's modified Eagle's medium.

$\mathrm{Ca}^{2+}$ remained at a significantly lower level following $3 \mathrm{~h}$ preservation in cardiomyocytes treated with different luteolin concentrations compared with the UW solution control group (all $\mathrm{P}<0.01$; Figs. 3A and C-E, and 4). When preserved for $6 \mathrm{~h}$ in UW solution supplemented with $7.5 \mu \mathrm{mol} / 1$ luteolin, cardiomyocytes exhibited $\mathrm{Ca}^{2+}$ accumulation but remained slightly lower than that in the UW solution group, although this difference was not observed to be significant. The 15 and $30 \mu \mathrm{mol} / 1$ luteolin groups continued to effectively prevent $\mathrm{Ca}^{2+}$ overload in the mitochondria, as the $\mathrm{Ca}^{2+}$ accumulation was significantly decreased compared with that in the UW solution control group (both $\mathrm{P}<0.01$; Figs. $3 \mathrm{~A}, \mathrm{D}$ and $\mathrm{E}$, and 4). When the concentration of luteolin was increased from 7.5 to $15 \mu \mathrm{mol} / 1$, there was a significant decrease in mitochondria calcium $(\mathrm{P}<0.01)$, most markedly following $6 \mathrm{~h}$ preservation (Fig. 4). However, the opposite effect was observed when the concentration of luteolin was increased from 15 to $30 \mu \mathrm{mol} / 1$ following 3 and $6 \mathrm{~h}$ preservation, suggesting a side effect of using high concentration luteolin for long-term preservation. (Fig. 4).

Luteolin suppresses SR calcium overload in rat cardiomyocytes during hypothermic preservation. The $\mathrm{Ca}^{2+}$ concentration changes in the SR of cardiomyocytes during the $6 \mathrm{~h}$ cold preservation using the same solutions was then investigated (Fig. 5). Similar to the $\mathrm{Ca}^{2+}$ accumulation in the mitochondria, the $\mathrm{Ca}^{2+}$ concentration inside the SR accumulated over time when cardiomyocytes were preserved in UW, and this accumulation was significantly exacerbated in the DMEM group at all three time points $(\mathrm{P}<0.01$; Figs. $5 \mathrm{~A}$ and $\mathrm{B}$, and 6). The $\mathrm{Ca}^{2+}$ accumulation inside the $\mathrm{SR}$ remained at a significantly decreased level in cardiomyocytes preserved in UW solution with 15 or $30 \mu \mathrm{mol} / 1$ luteolin throughout the $6 \mathrm{~h}$ preservation period compared with the UW solution control group ( $\mathrm{P}<0.01$; Figs. 5A and D, and 6). Even though cardiomyocytes preserved in UW solution with $7.5 \mu \mathrm{mol} / 1$ luteolin started to build up intracellular calcium slightly in the SR after $6 \mathrm{~h}$, there was no significant difference between all three time points (Fig. 6). In addition, compared with the UW solution control group, $\mathrm{Ca}^{2+}$ concentration was significantly decreased in the $7.5 \mu \mathrm{mol} / 1$ luteolin group at all three time points (all $\mathrm{P}<0.01$; Figs. 5A and $\mathrm{C}$, and 6). Together, these results indicate that adding luteolin to UW solution to preserve heart myocytes may inhibit intracellular calcium overload in the SR.

Luteolin represses CaM and MCU accumulation in rat cardiomyocytes during hypothermic preservation. The effect of luteolin supplementation on calcium signaling-related proteins during preservation was investigated. CaM concentration in rat cardiomyocytes during $6 \mathrm{~h}$ hypothermic preservation was determined first (Fig. 7). Myocytes preserved with DMEM in the control group expressed a significantly higher $\mathrm{CaM}$ compared with UW solution $(\mathrm{P}<0.01)$. All three groups of cardiomyocytes preserved in UW solution supplemented with different doses of luteolin had a significantly lower CaM concentration compared with the UW solution control group (all $\mathrm{P}<0.01$; Fig. 7A). The higher the dose of luteolin used to treat cardiomyocytes, the lower the CaM concentration throughout the $6 \mathrm{~h}$ preservation period. The differences between luteolin treatment groups at each time point were significant $(\mathrm{P}<0.01)$ except for cardiomyocytes preserved with 15 and $30 \mu \mathrm{mol} / 1$ luteolin during the $6 \mathrm{~h}$ preservation period (Fig. 7A). The differences between the CaM concentration of cardiomyocytes preserved in the UW solution control group at $1 \mathrm{~h}$ and the cardiomyocytes preserved in UW solution supplemented with $30 \mu \mathrm{mol} / 1$ luteolin for $6 \mathrm{~h}$, or cardiomyocytes preserved in UW solution supplemented with $15 \mu \mathrm{mol} / \mathrm{l}$ luteolin for $5 \mathrm{~h}$ were not significant. This suggests that the presence of luteolin during hypothermic preservation may repress $\mathrm{CaM}$ accumulation in cardiomyocytes and thus prolong preservation time.

Luteolin had an effect on limiting the accumulation of $\mathrm{Ca}^{2+}$ in the mitochondria, therefore, it was hypothesized that MCU, which is a vital transporter for mitochondria $\mathrm{Ca}^{2+}$ entry, may be decreased during luteolin preservation to suppress $\mathrm{Ca}^{2+}$ influx into the mitochondria. MCU concentration was determined by ELISA and the results indicated that luteolin supplementation at all doses reduced MCU levels compared with the UW solution control group (all $\mathrm{P}<0.01$; Fig. 7B). Compared with DMEM, utilizing UW solution reduced MCU expression over the $6 \mathrm{~h}$ preservation period, whereas luteolin supplemented UW solution further significantly reduced the MCU content in a dose dependent manner, from 3-6 h, for luteolin treated cardiomyocytes (all $\mathrm{P}<0.01$; Fig. 7B). The concentration of MCU was significantly lower following the $6 \mathrm{~h}$ preservation period with $30 \mu \mathrm{mol} / 1$ luteolin compared with $1 \mathrm{~h}$ preservation in the UW solution control group $(\mathrm{P}<0.01)$. However, no significant difference was observed between myocytes preserved with $15 \mu \mathrm{mol} / 1$ luteolin for $5 \mathrm{~h}$ and the UW solution group following $1 \mathrm{~h}$ preservation. The results imply that cold preservation of cardiomyocytes in UW solution supplemented with luteolin results in decreased MCU concentration, which serves a role in restricting mitochondrial $\mathrm{Ca}^{2+}$ influx.

Luteolin suppresses PKA activity and enhances $\mathrm{Ca}^{2+}-\mathrm{Mg}^{2+}$-ATPase activity during cold preservation. The role of PKA in luteolin prolonged heart preservation was investigated by measuring PKA enzymatic activity, as PKA is known to phosphorylate calcium regulatory proteins, including phospholamban (PLB) and ryanodine receptor (RyR) 2 (23,24). Cardiomyocytes preserved in UW solution supplemented with different doses of luteolin exhibited a reduction in PKA activity compared with the UW solution 


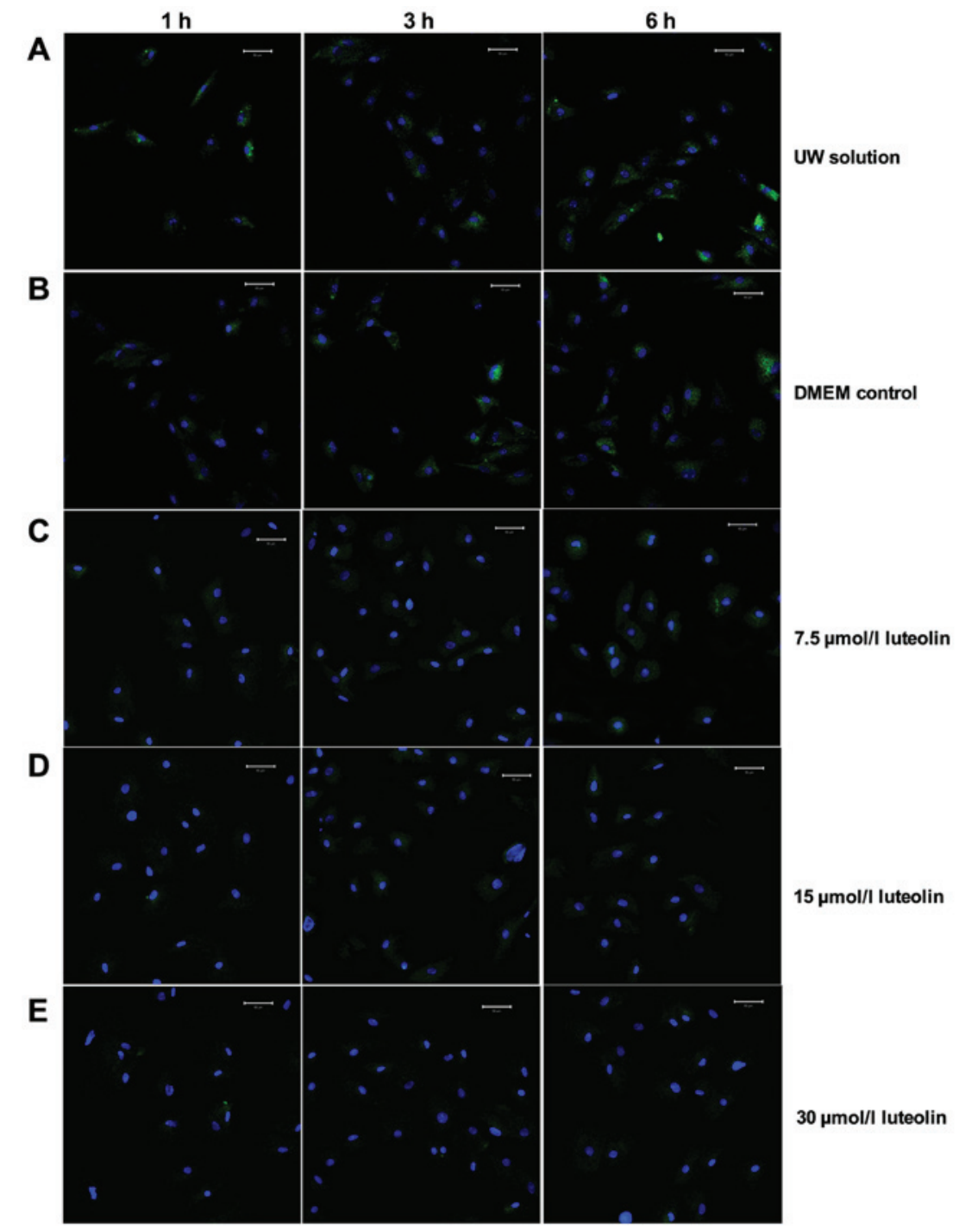

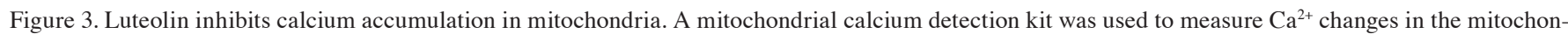
dria of cardiomyocyte preserved in the following solutions: (A) UW solution; (B) DMEM; (C) $7.5 \mu \mathrm{mol} / 1$ luteolin supplemented UW solution; (D) $15 \mu$ mol/1 luteolin supplemented UW solution and (E) $30 \mu \mathrm{mol} / 1$ luteolin supplemented UW solution. Representative multiphoton microscopy images from indicated time points were captured over the hypothermic preservation period. Scale bar, $50 \mu \mathrm{m}$. Green fluorescence indicates fluo-3 labeled $\mathrm{Ca}^{2+}$ while blue fluorescence indicates stained nuclei. UW solution, University of Wisconsin solution; DMEM, Dulbecco's modified Eagle's medium.

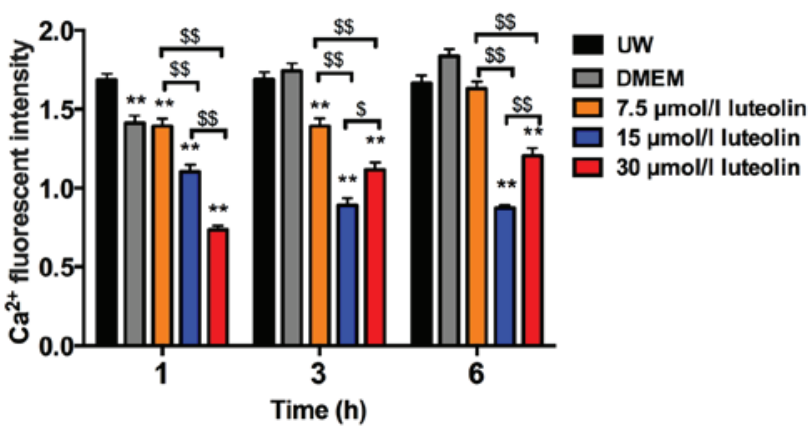

Figure 4. Fluorescence intensity of mitochondrial $\mathrm{Ca}^{2+}$ in the different groups. Data are presented as the mean \pm standard error of the mean. Experiments were performed in triplicate. ${ }^{* *} \mathrm{P}<0.01$ vs. UW solution group. ${ }^{\$ \$} \mathrm{P}<0.01$, ${ }^{\mathrm{s}} \mathrm{P}<0.05$. UW solution, University of Wisconsin solution; DMEM, Dulbecco's modified Eagle's medium.

control group (Fig. 7C); however, unlike MCU and CaM, cardiomyocytes treated with $7.5 \mu \mathrm{mol} / 1$ luteolin exhibited the biggest reduction compared with cardiomyocytes treated with 15 and $30 \mu \mathrm{mol} / 1$ luteolin (Fig. 7C). Nevertheless, even in cardiomyocytes treated with $30 \mu \mathrm{mol} / 1$ luteolin, which may have a toxic effect on PKA activity, the PKA activity was significantly decreased compared with the UW solution control group at $1-2 \mathrm{~h}(\mathrm{P}<0.01$; Fig. $7 \mathrm{C})$. Compared with the UW solution control group, PKA was significantly decreased in the 7.5 and $15 \mu \mathrm{mol} / 1$ luteolin treatment groups at all time points $(\mathrm{P}<0.01)$. When cardiomyocytes were preserved in DMEM, PKA activity was significantly increased compared with the UW solution control group $(\mathrm{P}<0.01)$, while adding luteolin to UW solution further suppressed PKA activity.

$\mathrm{Ca}^{2+}-\mathrm{Mg}^{2+}-$ ATPase activity was measured using ELISA. The results indicated that luteolin supplementation increased $\mathrm{Ca}^{2+}-\mathrm{Mg}^{2+}$-ATPase activity, with the most marked differences compared with the UW control group observed with 15 and $30 \mu \mathrm{mol} / 1$ luteolin treatment, and significant differences at 4-6 h $(\mathrm{P}<0.01$; Fig. 7D). There was a significant increase in $\mathrm{Ca}^{2+}-\mathrm{Mg}^{2+}$-ATPase activity when comparing the 


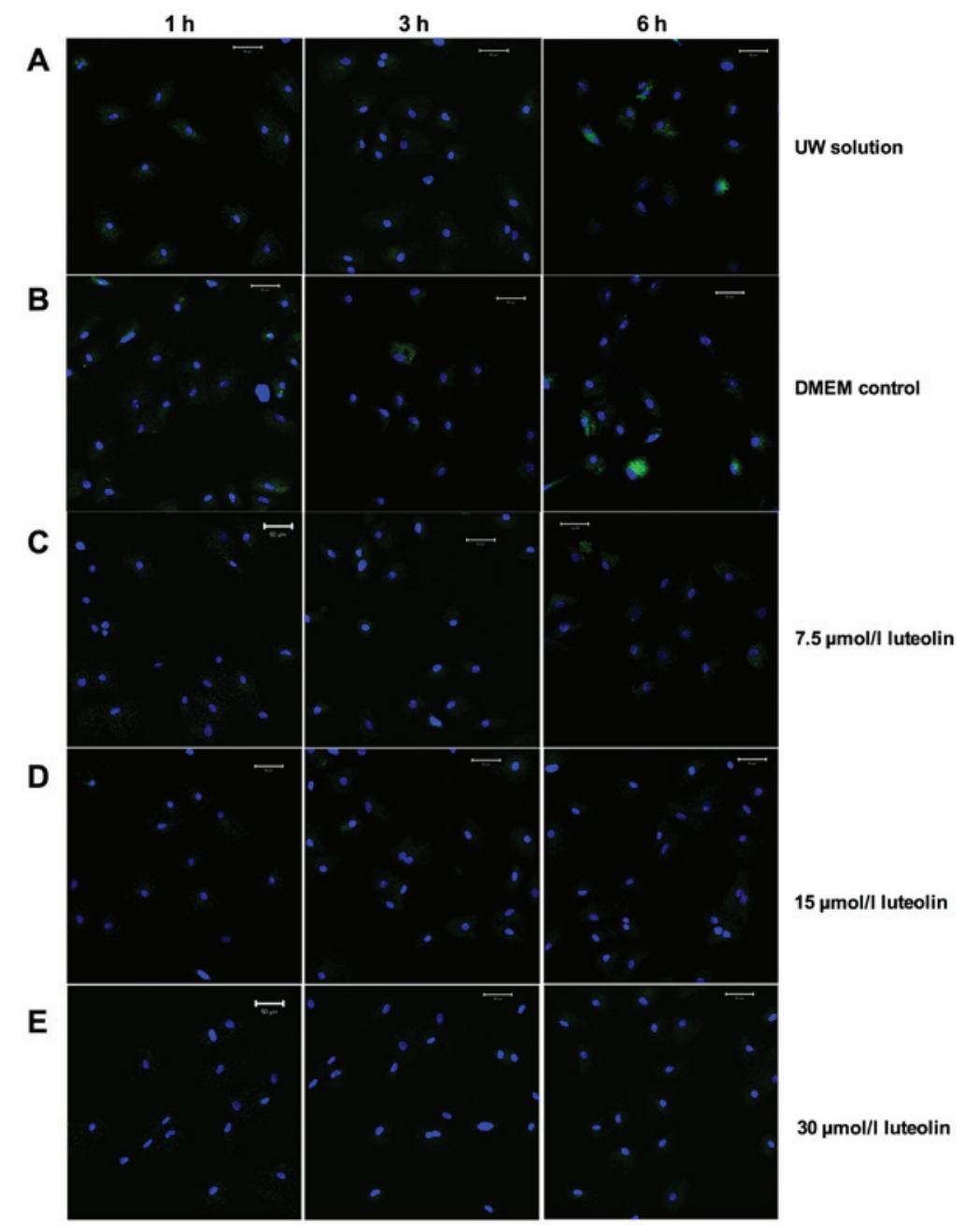

Figure 5. Luteolin suppresses calcium overload in the SR. An SR calcium kit was used to detect $\mathrm{Ca}^{2+}$ influx in the SR in myocytes preserved in the following solutions: (A) UW solution; (B) DMEM; (C) $7.5 \mu \mathrm{mol} / 1$ luteolin supplemented UW solution; (D) $15 \mu \mathrm{mol} / 1$ luteolin supplemented UW solution; and (E) $30 \mu \mathrm{mol} / 1$ luteolin supplemented UW solution. Representative multiphoton microscopy images from indicated time points were captured over the hypothermic preservation period. Scale bar, $50 \mu \mathrm{m}$. Green fluorescence indicates fluo-3 labeled $\mathrm{Ca}^{2+}$, while blue fluorescence indicates stained nuclei. UW solution, University of Wisconsin solution; DMEM, Dulbecco's modified Eagle's medium; SR, sarcoplasmic reticulum.

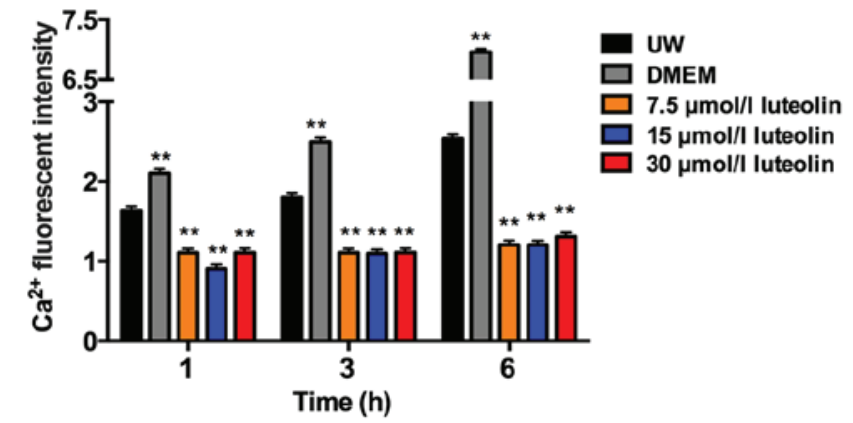

Figure 6. Fluorescence intensity of $\mathrm{Ca}^{2+}$ in the SR in different groups. Data are presented as the mean \pm standard error of the mean. Experiments were performed in triplicate. ${ }^{* *} \mathrm{P}<0.01$ vs. UW solution group. UW solution, University of Wisconsin solution; DMEM, Dulbecco's modified Eagle's medium; SR, sarcoplasmic reticulum.

response in cardiomyocytes treated with 7.5 and $15 \mu \mathrm{mol} / 1$ at 1,4 and $5 \mathrm{~h}(\mathrm{P}<0.01)$, however, there was no difference in $\mathrm{Ca}^{2+}-\mathrm{Mg}^{2+}$-ATPase activity when comparing cardiomyocytes preserved with 15 to $30 \mu \mathrm{mol} / 1$ luteolin at the different time points during the $6 \mathrm{~h}$ preservation period.

\section{Discussion}

The current accepted method of preservation for donor hearts is to cold preserve them in UW solution for 4-6 h prior to transplantation (4). However, short-term preservation limits the feasibility of transplantation surgery when cytotoxic events are initiated in cardiomyocytes, including the accumulation of ROS and $\mathrm{Ca}^{2+}$, which eventually results in cell death (25-27). The results of the present study demonstrated that hypothermia may induce $\mathrm{Ca}^{2+}$ accumulation in the cytosol, mitochondria and SR. Using a guinea pig heart as a model, a previous study indicated that cardioplegia may decrease cytosolic calcium accumulation and lead to better cardiac function following reperfusion (25). This suggests that suppression of $\mathrm{Ca}^{2+}$ overload during preservation may provide better protection to the heart and prolong storage time. The current study demonstrates the protective role of the flavonoid compound luteolin as an additive to UW solution, in modulating calcium cycling during hypothermal preservation of rat cardiomyocytes. Luteolin exists widely in plants, including peanut and chrysanthemum (28). The cardioprotective role of luteolin has been established in rabbit and rat hearts associated with 
A

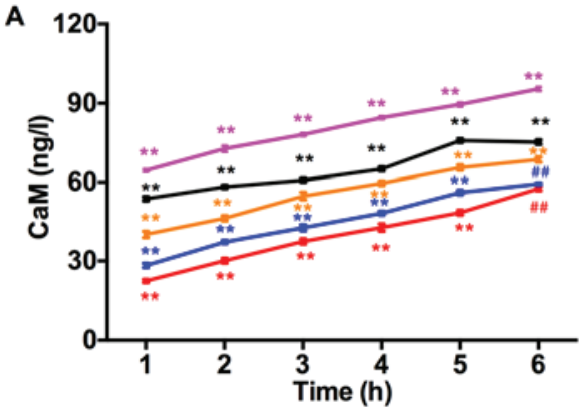

C

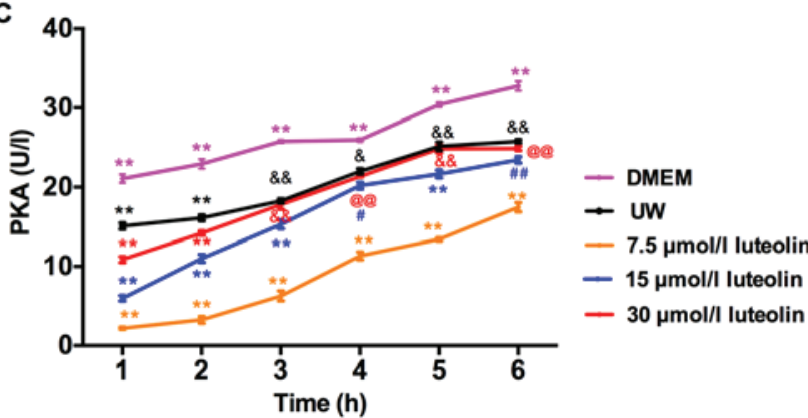

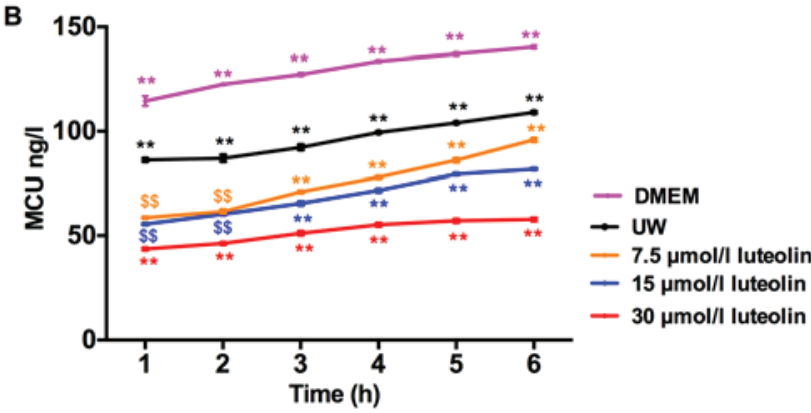

D

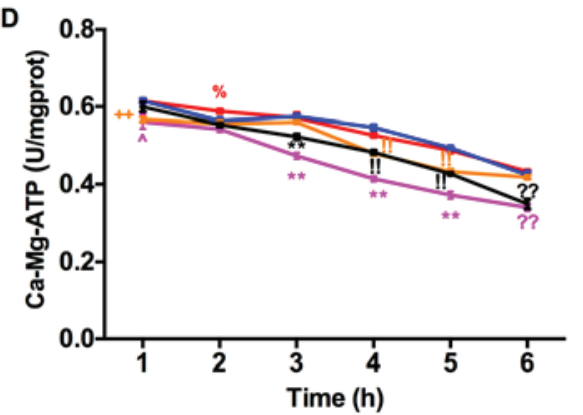

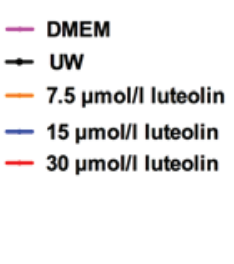

Figure 7. Luteolin exerts a cardioprotective effect on inhibiting the changes of intracellular calcium regulatory proteins, including MCU, CaM, PKA and $\mathrm{Ca}^{2+}-\mathrm{Mg}^{2+}$-ATPase during hypothermic preservation. ELISA was used to determine the concentration of (A) CaM and (B) MCU, and the activity of (C) PKA and (D) $\mathrm{Ca}^{2+}-\mathrm{Mg}^{2+}$-ATPase in myocytes during the $6 \mathrm{~h}$ preservation with DMEM (magenta line), UW solution (black line), $7.5 \mu \mathrm{mol} / 1$ luteolin (orange line), $15 \mu \mathrm{mol} / 1$ luteolin (blue line) and $30 \mu \mathrm{mol} / 1$ luteolin (red line), respectively. Data are presented as the mean \pm standard error of the mean. Experiments were performed in triplicate. ${ }^{* *} \mathrm{P}<0.01$ vs. all other groups; ${ }^{\# \#} \mathrm{P}<0.01$ vs. DMEM, UW and $7.5 \mu \mathrm{mol} / 1$ luteolin; ${ }^{\$ \$} \mathrm{P}<0.01$ vs. DMEM, UW and $30 \mu \mathrm{mol} / 1$ luteolin; \&\& $\mathrm{P}<0.01$ vs. DMEM, and 7.5 and $15 \mu \mathrm{mol} / 1$ luteolin; ${ }^{\%} \mathrm{P}<0.05$ vs. UW and DMEM; "P<0.01 vs. DMEM, and 15 and $30 \mu \mathrm{mol} / 1$ luteolin; ${ }^{@} @ \mathrm{P}<0.01$ vs. DMEM and $7.5 \mu \mathrm{mol} / 1$ luteolin; ${ }^{\wedge} \mathrm{P}<0.05$ vs. $\mathrm{UW}$, and 15 and $30 \mu \mathrm{mol} / 1$ luteolin; ${ }^{++} \mathrm{P}<0.01$ vs. 15 and $30 \mu \mathrm{mol} / 1$ luteolin. ${ }^{2} \mathrm{P}<0.01$ vs. UW, $7.5 \mu \mathrm{mol} / 1,15$ and 30 $\mu \mathrm{mol} / 1$ luteolin. UW, University of Wisconsin solution; DMEM, Dulbecco's modified Eagle's medium; ns, not significant; $\mathrm{MCU}$, mitochondria Ca ${ }^{2+}$ uniporter; CaM, calmodulin; PKA, protein kinase A.

ischemia $(8,29)$. However, to the best of our knowledge, there are no reports on the role served by luteolin in heart preservation and calcium signaling of cardiomyocytes. Our previous work demonstated that supplementation of luteolin into UW solution effectively inhibits the L-type calcium currents in hypoxia treated rat cardiomyocytes and provides better protection for rat hearts following 12 or $18 \mathrm{~h}$ hypothermic preservation in terms of contractile and diastolic function, enhancing coronary flow and preventing creatine kinase leakage (10). Consistent with this, the results of the current study demonstrated the role of luteolin in directly ameliorating intracellular $\mathrm{Ca}^{2+}$ overload through modulating regulatory proteins, including CaM, PKA, MCU and $\mathrm{Ca}^{2+}-\mathrm{Mg}^{2+}$-ATPase during a $6 \mathrm{~h}$ preservation period.

Calcium serves a critical role in regulating cardiomyocyte function including contraction, transcription regulation and cell death $(14,23)$. Hypothermia induces intracellular $\mathrm{Ca}^{2+}$ accumulation, which may promote the activation of $\mathrm{Ca}^{2+}$-dependent proteases, leading to the apoptosis and necrosis of cardiomyocytes (26). Calcium ion influx into cardiomyocytes through a $\mathrm{Ca}^{2+}$ current is dependent on L-type voltage-operated calcium channels, which are regulated by the activation of $\beta$-AR, downstream PKA and $\mathrm{Na}^{+} / \mathrm{Ca}^{2+}$ exchange (NCX) $(23,30)$. The major pathway used by calcium to exit cells is through plasma membrane NCX and the $\mathrm{Ca}^{2+}-\mathrm{Mg}^{2+}$-ATPase pump. The SR and mitochondria are also critical for the removal of cytosolic calcium. Cytosolic $\mathrm{Ca}^{2+}$ is transported through the MCU into the mitochondria, while SR Ca-ATPase (SERCA) mediates the majority of calcium ion uptake $(14,23)$. Using a fluorescent probe to detect $\mathrm{Ca}^{2+}$ in the current study, confocal microscopy data indicated that $\mathrm{Ca}^{2+}$ was decreased in the cytosol, mitochondria and SR during the $6 \mathrm{~h}$ cold preservation period with luteolin-supplemented UW solution. Furthermore, the $\mathrm{Ca}^{2+}$ load in the cytosol, mitochondria and SR of cardiomyocytes following $6 \mathrm{~h}$ preservation with luteolin was decreased compared with that in those preserved with UW solution alone for $1 \mathrm{~h}$. There was no significant accumulation of $\mathrm{Ca}^{2+}$ in SR over time observed in the cardiomyocytes preserved with UW solution supplemented with different doses of luteolin during $6 \mathrm{~h}$ preservation. By contrast, $\mathrm{Ca}^{2+}$ accumulated in the SR over time in the UW solution control group. Even though the dose response was not significant between the luteolin-treated groups in preventing intracellular $\mathrm{Ca}^{2+}$ overload, supplementing luteolin to cardiomyocytes was able to effectively suppress $\mathrm{Ca}^{2+}$ accumulation in the SR which stayed at a basal level of $\mathrm{Ca}^{2+}$ concentration at the end of the $6 \mathrm{~h}$ preservation period.

Hypothermia-induced mitochondria $\mathrm{Ca}^{2+}$ accumulation is harmful to mitochondrial functions, including the generation of ATP (25). Suppression of mitochondrial $\mathrm{Ca}^{2+}$ overload may ameliorate abnormal function in the mitochondria following reperfusion (31). A recent study also demonstrated the inhibition of MCU, which is responsible for mitochondrial $\mathrm{Ca}^{2+}$ uptake and prevents mitophagy in neurocytes following ischemia/reperfusion injury (13). In the current study, during hypothermic preservation with luteolin-supplemented UW solution, upregulation of MCU over time was significantly 
delayed in cardiomyocytes. Bonferroni post-hoc comparison indicated that myocytes preserved for $5 \mathrm{~h}$ in $7.5 \mu \mathrm{mol} / 1$ luteolin supplemented UW solution expressed similar MCU to those preserved for $1 \mathrm{~h}$ with UW solution only. Delayed MCU increase was extended to $6 \mathrm{~h}$ in the two groups preserved with higher concentrations of luteolin. This further indicated the protective role of luteolin in slowing hypothermia-induced dysfunction in cardiomyocytes.

Besides mitochondria, the importance of the SR in modulating calcium homeostasis in cardiomyocytes has been established previously. The SR absorbs $\mathrm{Ca}^{2+}$ through SERCA, which requires energy consumption and is regulated through the phosphorylation of PLB and the release of $\mathrm{Ca}^{2+}$ through the RyR (14). PKA, $\mathrm{Ca}^{2+}-\mathrm{Mg}^{2+}$-ATPase and $\mathrm{CaM}$ are involved in this SR mediated calcium exchanging. The activation of PKA, which is mediated through an increasing level of cAMP, modulates L-type calcium channel opening $(32,33)$. In the current study, it was demonstrated that luteolin inhibits PKA activity in cardiomyocytes preserved with luteolin. This inhibition may restrict L-type calcium channel opening and thereby L-type calcium currents. Furthermore, PLB is polymerized through phosphorylation mediated by PKA. Polymerized PLB is unable to interact with SERCA and results in $\mathrm{Ca}^{2+}$ accumulation in the SR $(34,35)$. Thus, suppression of PKA activity by luteolin may lead to the depolymerization of PLB, which decreases pump activity and subsequently inhibits $\mathrm{Ca}^{2+}$ influx into the SR (23). CaM is a calcium binding protein that regulates $\mathrm{Ca}^{2+}$ efflux via RyR and Ca-dependent inactivation of the L-type calcium current (14). The results of the ELISAs performed in the current study indicated that luteolin decreased $\mathrm{CaM}$ concentration and delayed its accumulation in cardiomyocytes during cold storage in a dose-dependent manner. Free calcium ions from the cytosol bind to form a $\mathrm{Ca}-\mathrm{CaM}$ complex, therefore the decreased $\mathrm{CaM}$ concentration in myocytes preserved in luteolin supplemented UW solution may be due to the enhanced interaction between $\mathrm{Ca}-\mathrm{CaM}$, which inactivates the L-type calcium channels and decelerates $\mathrm{Ca}^{2+}$ influx (36). This increased $\mathrm{Ca}-\mathrm{CaM}$ formation may also consume the cytosolic $\mathrm{Ca}^{2+}$ and subsequently decrease $\mathrm{Ca}^{2+}$ accumulation. As a critical enzyme in the SR that participates in $\mathrm{Ca}^{2+}$ influx, $\mathrm{Ca}^{2+}-\mathrm{Mg}^{2+}$-ATPase positively regulates the transportation of $\mathrm{Ca}^{2+}$ across the cell membrane via the hydrolysis of ATP (37). The current study indicated that there was enhanced $\mathrm{Ca}^{2+}-\mathrm{Mg}^{2+}$-ATPase activity in cardiomyocytes preserved in UW solution supplemented with luteolin, although the dose effect was only observed between cardiomyocytes preserved with 7.5 and $15 \mu \mathrm{mol} / 1$ luteolin-supplemented UW solution. The deactivation of $\mathrm{Ca}^{2+}-\mathrm{Mg}^{2+}-$ ATPase is exacerbated during hypothermic preservation, leading to intracellular calcium accumulation through modulating SR Ca ${ }^{2+}$ uptake (19). However, the results of the current study suggest that the addition of luteolin to UW solution to preserve cardiomyocytes may repress this process and ameliorate $\mathrm{Ca}^{2+}$ overload. The activity of $\mathrm{Ca}^{2+}-\mathrm{Mg}^{2+}$-ATPase following $3 \mathrm{~h}$ preservation with the three different doses of luteolin was similar to that of cardiomyocytes preserved with UW solution for $1 \mathrm{~h}$, which indicates that luteolin serves a protective role in the extension of rat heart preservation. This novel effect of luteolin on cardiomyocyte calcium cycling may shed new light on extending heart hypothermic preservation.
In conclusion, there were decreased concentrations of MCU and CaM, decreased PKA enzyme activity and enhanced $\mathrm{Ca}^{2+}-\mathrm{Mg}^{2+}$-ATPase activity in the cardiomyocytes preserved with luteolin and UW solution for $6 \mathrm{~h}$. These results indicate that luteolin may reduce intracellular $\mathrm{Ca}^{2+}$ through restrained $\mathrm{Ca}^{2+}$ influx and enhanced $\mathrm{Ca}^{2+}$ extruding. The results indicated that intracellular $\mathrm{Ca}^{2+}$ overload in rat cardiomyocytes was suppressed by the addition of luteolin. Together, the alteration of $\mathrm{Ca}^{2+}$ homeostasis-regulating proteins by luteolin may be the reason why it inhibits intracellular $\mathrm{Ca}^{2+}$ accumulation and subsequently prolongs heart preservation. This novel effect of luteolin on cardiomyocyte calcium cycling may shed new light on extending heart hypothermic preservation. Adding luteolin to UW solution to preserve the heart may extend the preservation time which may greatly facilitate clinical heart transplantation.

\section{Acknowledgements}

This study was supported by a grant from the Natural Science Foundation of China (grant no. 81360029) and Natural Science Foundation of Hainan Province (grant no. 809042). The authors would like to thank BersinBio (GuangZhou, China) for their technical assistance.

\section{References}

1. Alsoufi B, Deshpande S, McCracken C, Kogon B, Vincent R, Mahle W and Kanter K: Outcomes and risk factors for heart transplantation in children with congenital heart disease. J Thorac Cardiovasc Surg 150: 1455-1462.e3, 2015.

2. Vitali E, Colombo T, Fratto P, Russo C, Bruschi G and Frigerio M: Surgical therapy in advanced heart failure. Am J Cardiol 91: 88F-94F, 2003.

3. Colvin M, Smith JM, Skeans MA, Edwards LB, Uccellini K, Snyder JJ, Israni AK and Kasiske BL: OPTN/SRTR 2015 annual data report: Heart. Am J Transplant 17 (Suppl 1): S286-S356, 2017.

4. Belzer FO and Southard JH: Principles of solid-organ preservation by cold storage. Transplantation 45: 673-676, 1988.

5. Jahania MS, Sanchez JA, Narayan P, Lasley RD and Mentzer RM Jr: Heart preservation for transplantation: Principles and strategies. Ann Thorac Surg 68: 1983-1987, 1999.

6. Benavente-García O and Castillo J: Update on uses and properties of citrus flavonoids: New findings in anticancer, cardiovascular, and anti-inflammatory activity. J Agric Food Chem 56: 6185-6205, 2008.

7. Choi JH, Kim YS, Shin CH, Lee HJ and Kim S: Antithrombotic activities of luteolin in vitro and in vivo. J Biochem Mol Toxicol 29: 552-558, 2015.

8. Qi L, Pan H, Li D, Fang F, Chen D and Sun H: Luteolin improves contractile function and attenuates apoptosis following ischemia-reperfusion in adult rat cardiomyocytes. Eur J Pharmacol 668: 201-207, 2011.

9. Lin Y, Shi R, Wang X and Shen HM: Luteolin, a flavonoid with potential for cancer prevention and therapy. Curr Cancer Drug Targets 8: 634-646, 2008.

10. Yan QF, Yan GF and Yang DK: Myocardial protective effects of luteolin on isolated rat heart in hypothermic preservation. Zhongguo Ying Yong Sheng Li Xue Za Zhi 28: 154-158, 2012 (In Chinese).

11. Murgia M, Giorgi C, Pinton P and Rizzuto R: Controlling metabolism and cell death: At the heart of mitochondrial calcium signalling. J Mol Cell Cardiol 46: 781-788, 2009.

12. Dorn GW II and Maack C: SR and mitochondria: Calcium cross-talk between kissing cousins. J Mol Cell Cardiol 55: 42-49, 2013.

13. Yu S, Zheng S, Leng J, Wang S, Zhao $\mathrm{T}$ and Liu J: Inhibition of mitochondrial calcium uniporter protects neurocytes from ischemia/reperfusion injury via the inhibition of excessive mitophagy. Neurosci Lett 628: 24-29, 2016. 
14. Fearnley CJ, Roderick HL and Bootman MD: Calcium signaling in cardiac myocytes. Cold Spring Harb Perspect Biol 3: a004242, 2011.

15. Bers DM: Cardiac excitation-contraction coupling: Nature 415: 198-205, 2002.

16. Balshaw DM, Yamaguchi N and Meissner G: Modulation of intracellular calcium-release channels by calmodulin. J Membr Biol 185: 1-8, 2002.

17. Lanner JT, Georgiou DK, Joshi AD and Hamilton SL: Ryanodine receptors: Structure, expression, molecular details, and function in calcium release. Cold Spring Harb Perspect Biol 2: a003996, 2010.

18. Nakamura J: $\mathrm{pH}$ and temperature resolve the kinetics of two pools of calcium bound to the sarcoplasmic reticulum $\mathrm{Ca} 2+$ -ATPase. J Biol Chem 264: 17029-17031, 1989.

19. Morris GL, Cheng HC, Colyer J and Wang JH: Phospholamban regulation of cardiac sarcoplasmic reticulum $(\mathrm{Ca}(2+)-\mathrm{Mg} 2+)$-ATPase. Mechanism of regulation and site of monoclonal antibody interaction. J Biol Chem 266: 11270-11275, 1991.

20. National Research Council: Guide for the Care and Use of Laboratory Animals. 8th edition. National Academy Press, Washington, DC, 2011.

21. Louch WE, Sheehan KA and Wolska BM: Methods in cardiomyocyte isolation, culture, and gene transfer. J Mol Cell Cardiol 51: 288-298, 2011.

22. Bell RM, Mocanu MM and Yellon DM: Retrograde heart perfusion: The Langendorff technique of isolated heart perfusion. J Mol Cell Cardiol 50: 940-950, 2011.

23. Bers DM: Calcium cycling and signaling in cardiac myocytes. Annu Rev Physiol 70: 23-49, 2008.

24. Müller BA and Dhalla NS: Mechanisms of the beneficial actions of ischemic preconditioning on subcellular remodeling in ischemic-reperfused heart. Curr Cardiol Rev 6: 255-264, 2010.

25. Stowe DF, Varadarajan SG, An J and Smart SC: Reduced cytosolic $\mathrm{Ca}(2+)$ loading and improved cardiac function after cardioplegic cold storage of guinea pig isolated hearts. Circulation 102: $1172-1177,2000$

26. Wakayama K, Fukai M, Yamashita K, Kimura T, Hirokata G, Shibasaki S, Fukumori D, Haga S, Sugawara M, Suzuki T, et al: Successful transplantation of rat hearts subjected to extended cold preservation with a novel preservation solution. Transpl Int 25: 696-706, 2012

27. Riess ML, Camara AK, Kevin LG, An J and Stowe DF: Reduced reactive $\mathrm{O} 2$ species formation and preserved mitochondrial $\mathrm{NADH}$ and $[\mathrm{Ca} 2+]$ levels during short-term 17 degrees $\mathrm{C}$ ischemia in intact hearts. Cardiovasc Res 61: 580-590, 2004.
28. Harnly JM, Doherty RF, Beecher GR, Holden JM, Haytowitz DB, Bhagwat S and Gebhardt S: Flavonoid content of U.S. fruits, vegetables, and nuts. J Agric Food Chem 54: 9966-9977, 2006.

29. Rump AF, Schüssler M, Acar D, Cordes A, Ratke R, Theisohn M, Rösen R, Klaus W and Fricke U: Effects of different inotropes with antioxidant properties on acute regional myocardial ischemia in isolated rabbit hearts. Gen Pharmacol 26: 603-611, 1995.

30. Weber CR, Piacentino V III, Houser SR and Bers DM: Dynamic regulation of sodium/calcium exchange function in human heart failure. Circulation 108: 2224-2229, 2003.

31. Miyamae M, Camacho SA, Weiner MW and Figueredo VM: Attenuation of postischemic reperfusion injury is related to prevention of $[\mathrm{Ca} 2+] \mathrm{m}$ overload in rat hearts. Am J Physiol 271: H2145-H2153, 1996.

32. Fusi F, Manetti F, Durante M, Sgaragli G and Saponara S: The vasodilator papaverine stimulates L-type $\mathrm{Ca}(2+)$ current in rat tail artery myocytes via a PKA-dependent mechanism. Vascul Pharmacol 76: 53-61, 2016.

33. Catterall WA: Structure and regulation of voltage-gated $\mathrm{Ca} 2+$ channels. Annu Rev Cell Dev Biol 16: 521-555, 2000.

34. Simmerman HK, Collins JH, Theibert JL, Wegener AD and Jones LR: Sequence analysis of phospholamban. Identification of phosphorylation sites and two major structural domains. J Biol Chem 261: 13333-13341, 1986.

35. Kimura Y, Kurzydlowski K, Tada M and MacLennan DH: Phospholamban inhibitory function is activated by depolymerization. J Biol Chem 272: 15061-15064, 1997.

36. Tang W, Halling DB, Black DJ, Pate P, Zhang JZ, Pedersen S, Altschuld RA and Hamilton SL: Apocalmodulin and Ca2+ calmodulin-binding sites on the CaV1.2 channel. Biophys J 85: $1538-1547,2003$.

37. Swanson JE, Lokesh BR and Kinsella JE: Ca2+-Mg2+ ATPase of mouse cardiac sarcoplasmic reticulum is affected by membrane n- 6 and n-3 polyunsaturated fatty acid content. J Nutr 119 : 364-372, 1989 .

This work is licensed under a Creative Commons Attribution-NonCommercial-NoDerivatives 4.0 International (CC BY-NC-ND 4.0) License. 